\title{
HILBERT-SAMUEL POLYNOMIALS FOR THE CONTRAVARIANT EXTENSION FUNCTOR
}

\author{
ANDREW CRABBE, DANIEL KATZ, \\ JANET STRIULI, AND EMANOIL THEODORESCU
}

\begin{abstract}
Let $(R, \mathfrak{m})$ be a local ring, and let $M$ and $N$ be finite $R$-modules. In this paper we give a formula for the degree of the polynomial giving the lengths of the modules $\operatorname{Ext}_{R}^{i}\left(M, N / \mathfrak{m}^{n} N\right)$. A number of corollaries are given, and more general filtrations are also considered.
\end{abstract}

\section{$\S 1$. Introduction}

Let $(R, \mathfrak{m}, \mathrm{k})$ be a Noetherian local ring, let $I \subseteq R$ be an ideal, and let $M$ and $N$ be finitely generated $R$-modules. It is well known that if the lengths $\lambda\left(M / I^{n} M\right)$ of the modules $M / I^{n} M$ are finite for $n$ large, these lengths are given by a rational polynomial of degree $\operatorname{dim}(M)$. In [7] (see also [6]) it is shown that the lengths of the modules $\operatorname{Tor}_{i}^{R}\left(M, N / I^{n} N\right)$ and $\operatorname{Ext}_{R}^{i}\left(M, N / I^{n} N\right)$ have polynomial growth for large $n$ whenever the lengths of these modules are finite. However, the degrees of the corresponding Hilbert-Samuel polynomials are not as easy to determine (see [7], [3], and [5]). This paper has three purposes. The first is to improve the known estimates for the degrees of the polynomials giving the lengths of $\operatorname{Ext}_{R}^{i}\left(M, N / I^{n} N\right)$ and $\operatorname{Tor}_{i}^{R}\left(M, N / I^{n} N\right)$ in the case $I=\mathfrak{m}$ by giving a precise formula for these degrees. Previous results for the case $I=\mathfrak{m}$ for the torsion functor were given in [3] and [5], where various assumptions were made in order to control this degree. In this paper we do not need to make any assumptions on $M, N$, or $R$ to obtain our formulas, and we need only make modest assumptions on them to obtain a formula that makes direct reference only to $M$ and $N$. In fact, in Section 2 we begin by giving a general

Received October 18, 2008. Revised August 14, 2009. Accepted August 17, 2009. 2000 Mathematics Subject Classification. Primary 13D07, 13D40, 13H15, 13 C11.

The third author was partially supported by National Science Foundation grant DMS0201904.

(C) 2010 by The Editorial Board of the Nagoya Mathematical Journal 
formula (see Proposition 2.1) for the degree of the Hilbert polynomial associated to general cohomology or homology modules, which specializes to our main results in Section 3 when the ideal in question is $\mathfrak{m}$ and the cohomology is determined by either the contravariant extension functor (Theorem 3.2) or the torsion functor (Theorem 3.12). For example, the following theorem is a direct consequence of our main result in Section 3 . Here we write $\mathcal{E}_{M, N, \mathfrak{m}}^{i}(n)$ for the Hilbert polynomial giving $\lambda\left(\operatorname{Ext}_{R}^{i}\left(N, M / \mathfrak{m}^{n} M\right)\right)$, for $n$ large.

Theorem 1.1. Let $(R, \mathfrak{m}, \mathrm{k})$ be a local Noetherian ring, and let $M$ and $N$ be two finitely generated $R$-modules such that $M$ has a rank and $\operatorname{dim}(N)=$ $\operatorname{dim}(R)$. Then $\operatorname{deg}\left(\mathcal{E}_{M, N, \mathfrak{m}}^{i}(n)\right)=\operatorname{dim}(N)-1$.

As we show below, the degree of $\mathcal{E}_{M, N, \mathfrak{m}}^{i}(n)$ is partially controlled by the dimension of $\Omega_{R}^{i}(M)$, the $i$ th syzygy of $M$. Consequently, as an application of our degree formula, we obtain the following proposition, which yields some information about the dimension of the syzygies of finite-length modules. In Proposition 1.2, we use p.d.( $M)$ to denote the projective dimension of $M$.

Proposition 1.2. Let $(R, \mathfrak{m}, \mathrm{k})$ be a local ring, and let $M$ be a finitely generated $R$-module, free of constant rank on the punctured spectrum of $R$. Assume that $\operatorname{dim}(R) \geq 2$, that the Betti numbers of $M$ are nondecreasing, and that $i<$ p.d. $(M)$. Then $\operatorname{dim}\left(\Omega_{R}^{i+1}(M)\right)=d$.

Our second purpose, especially regarding Section 3, is to lay the groundwork for results concerning indecomposable modules in [2], where knowledge of the relative growth of the Hilbert polynomials of large syzygies of the residue field $\mathrm{k}$ is required. In particular, Theorem 1.1 above plays a crucial role in [2].

Finally, in Section 4, we address our third purpose, to show that the results of Section 3 can, in many cases, be extended to more general filtrations to give results for the extension functor parallel to those given in [5] for the torsion functor.

\section{§2. General cohomology}

Throughout, $(R, \mathfrak{m}, \mathrm{k})$ denotes a Noetherian local ring of Krull dimension $d$, and all modules are finitely generated $R$-modules. In this section we prove a general result about the Hilbert polynomial associated to an ideal and the cohomology (or homology) of a complex. We start by letting

$$
\mathcal{C}: \quad X \stackrel{\alpha}{\rightarrow} Y \stackrel{\beta}{\rightarrow} Z
$$


be a complex of finitely generated $R$-modules with $Y \neq 0$. We assume that $I \subseteq R$ is an ideal such that the homology modules $\mathrm{H}\left(\mathcal{C} \otimes R / I^{n}\right)$ associated to $\mathcal{C} \otimes R / I^{n}$ have finite length for $n$ large. By [7, Proposition 3], the lengths of the modules $\mathrm{H}\left(\mathcal{C} \otimes R / I^{n}\right)$ are given by a polynomial $P_{I}^{\mathcal{C}}(n)$ for $n$ large. The following proposition strengthens [7, Proposition 3], in that we replace the degree estimate there by an equality. In the statement of this proposition we set

$$
\mathcal{M}:=\bigoplus_{n \geq 0}\left(I^{n} Z \cap \operatorname{im}(\beta)\right) / I^{n} \operatorname{im}(\beta) .
$$

Note that $\mathcal{M}$ is a finitely generated graded module over the Rees algebra of $R$ with respect to $I$, so that if its graded components have finite length as $R$-modules, then these lengths are ultimately given by a rational polynomial of degree $\operatorname{dim}(\mathcal{M})-1$.

Proposition 2.1. Let $(R, \mathfrak{m}, \mathrm{k})$ be a local ring, and let $\mathcal{C}$ as above be a complex of finitely generated $R$-modules with $Y \neq 0$. Let $I \subseteq R$ be an ideal such that the lengths of the cohomology modules $\mathrm{H}\left(\mathcal{C} \otimes R / I^{n}\right)$ are nonzero and finite for $n$ large, and let $P_{I}^{\mathcal{C}}(n)$ denote the corresponding HilbertSamuel polynomial. Then

$$
\operatorname{deg}\left(P_{I}^{\mathcal{C}}(n)\right)=\max \{\operatorname{dim}(\mathrm{H}(\mathcal{C})), \operatorname{dim}(\mathcal{M})-1\} .
$$

Proof. Set $A:=\operatorname{ker}(\beta)$ and $B:=\operatorname{im}(\alpha)$. We begin by arguing as in the proof of [7, Proposition 3]. By the Artin-Rees lemma, there exists $h>0$ so that, for $n \geq h$,

$$
I^{n} Z \cap \operatorname{im}(\beta)=I^{n-h}\left(I^{h} Z \cap \operatorname{im}(\beta)\right) .
$$

Since an element in the cohomology of the complex $\mathcal{C} \otimes R / I^{n}$ corresponds to an element in $Y$ that gets mapped by $\beta$ into $I^{n} Z \cap \operatorname{im}(\beta)$, it follows (see [7]) that for $n \geq h$,

$$
\mathrm{H}\left(\mathcal{C} \otimes R / I^{n}\right)=\frac{A+I^{n-h} C}{B+I^{n-h} D},
$$

where $C:=\beta^{-1}\left(I^{h} Z\right)$ and $D:=I^{h} Y$. Now for $n$ large,

$$
\begin{aligned}
P_{I}^{\mathcal{C}}(n) & =\lambda\left(\frac{A+I^{n-h} C}{B+I^{n-h} D}\right)=\lambda\left(\frac{A+I^{n-h} C}{A+I^{n-h} D}\right)+\lambda\left(\frac{A+I^{n-h} D}{B+I^{n-h} D}\right) \\
& =\lambda\left(\frac{A+I^{n-h} C}{A+I^{n-h} D}\right)+\lambda\left(\frac{U+I^{n-h} W}{I^{n-h} W}\right),
\end{aligned}
$$

where $U:=A / B=\mathrm{H}(\mathcal{C})$ and $W:=(D+B) / B$. We first note that by $[7$, Lemma 2], both length expressions on the right-hand sides of (2.1) and 
(2.2) are given by polynomials. Let $P_{1}(n)$ denote the polynomial giving the lengths of $\left(A+I^{n-h} C\right) /\left(A+I^{n-h} D\right)$, and let $P_{2}(n)$ denote the polynomial giving the lengths of $\left(U+I^{n-h} W\right) / I^{n-h} W$. We first calculate the degree of $P_{1}(n)$. For this, we note that, by definition,

$$
\frac{A+I^{n-h} C}{A+I^{n-h} D} \cong \frac{I^{n} Z \cap \operatorname{im}(\beta)}{I^{n} \operatorname{im}(\beta)} .
$$

Thus, by the definition of $\mathcal{M}, \operatorname{deg}\left(P_{1}(n)\right)=\operatorname{dim}(\mathcal{M})-1$ if $P_{1}(n)$ is not identically zero.

We now show that if $P_{2}(n)$ is not identically zero the degree of $P_{2}(n)$ equals $\operatorname{dim}(U)=\operatorname{dim}(\mathrm{H}(\mathcal{C}))$. Since $P_{I}^{\mathcal{C}}(n)=P_{1}(n)+P_{2}(n)$, this will complete the proof of the proposition. For this, note that there exists $c>0$ so that for $n$ sufficiently large,

$$
P_{2}(n)=\lambda\left(\frac{U}{U \cap I^{n-h} W}\right)=\lambda\left(\frac{U}{U \cap I^{c} W}\right)+\lambda\left(\frac{U \cap I^{c} W}{I^{n-h-c}\left(U \cap I^{c} W\right)}\right),
$$

so either $U \cap I^{c} W=0$ or $P_{2}(n)$ has degree equal to $\operatorname{dim}\left(U \cap I^{c} W\right)$. But since $U /\left(U \cap I^{c} W\right)$ has finite length if $U \cap I^{c} W \neq 0$, then $\operatorname{dim}(U)=\operatorname{dim}\left(U \cap I^{c} W\right)$, which gives what we want.

In the case $I=\mathfrak{m}$, we can replace $\mathcal{M}$ in the statement of Proposition 2.1 by $T:=\operatorname{im}(\beta)$. This allows us to give a precise formula for $P_{\mathfrak{m}}^{\mathcal{C}}(n)$ in terms of the modules appearing in the complex $\mathcal{C}$. Our main results in the next section concerning the extension and torsion functors are immediate consequences of the following theorem.

THEOREM 2.2. Let $(R, \mathfrak{m}, \mathrm{k})$ be a local ring, and let $\mathcal{C}$ as above be a complex of finitely generated $R$-modules with $Y \neq 0$. Write $P_{\mathfrak{m}}^{\mathcal{C}}(n)$ for the Hilbert-Samuel polynomial giving the lengths of the cohomology modules $\mathrm{H}\left(\mathcal{C} \otimes R / \mathfrak{m}^{n}\right)$, for $n$ large, and set $T:=\operatorname{im}(\beta)$. If $T \subseteq \mathfrak{m} Z$, then

$$
\operatorname{deg}\left(P_{\mathfrak{m}}^{\mathcal{C}}(n)\right)=\max \{\operatorname{dim}(\mathrm{H}(\mathcal{C})), \operatorname{dim}(T)-1\} .
$$

Proof. By Proposition 2.1, we have to show only that $\operatorname{dim}(\mathcal{M})=\operatorname{dim}(T)$. On the one hand, since $T \subseteq \mathfrak{m} Z$, we have

$$
\mathfrak{m}^{n-1} T \subseteq \mathfrak{m}^{n} Z \cap T .
$$

Therefore, $\bigoplus_{n \geq 1} \mathfrak{m}^{n-1} T / \mathfrak{m}^{n} T \subseteq \mathcal{M}$. It follows that

$$
\operatorname{dim}(T)=\operatorname{dim}\left(\bigoplus_{n \geq 1} \mathfrak{m}^{n-1} T / \mathfrak{m}^{n} T\right) \leq \operatorname{dim}(\mathcal{M}) .
$$


On the other hand, by the Artin-Rees lemma, there exists an $e \geq 1$ so that for $n$ large, $\mathfrak{m}^{n} Z \cap T=\mathfrak{m}^{n-e}\left(\mathfrak{m}^{e} Z \cap T\right)$. As in the proof of Proposition 2.1, write $P_{1}(n)$ for the polynomial giving the lengths of $\mathcal{M}_{n}$. Then

$$
P_{1}(n) \leq \lambda\left(T / \mathfrak{m}^{n} T\right)-\lambda\left(T / \mathfrak{m}^{n-e} T\right),
$$

and the degree of the polynomial giving the latter difference equals $\operatorname{dim}(T)-1$. This shows that $\mathcal{M}$ has dimension less than or equal to the dimension of $T$ and thus must have dimension equal to $T$, which is what we want.

In the following corollary, we record some observations related to the case that $P_{I}^{\mathcal{C}}(n)$ is the zero polynomial.

Corollary 2.3. Retain the notation from Proposition 2.1 and Theorem 2.2.

(a) If $P_{I}^{\mathcal{C}}(n) \equiv 0$, then $\mathrm{H}(\mathcal{C})=0$.

(b) If $\operatorname{im}(\alpha) \subseteq \mathfrak{m} Y$, then $\mathrm{H}(\mathcal{C})$ and $\operatorname{im}(\beta)$ cannot be simultaneously zero.

(c) If $\operatorname{im}(\alpha) \subseteq \mathfrak{m} Y$ and $\operatorname{im}(\beta) \subseteq \mathfrak{m} Z$, then $P_{\mathfrak{m}}^{\mathcal{C}}(n) \equiv 0$ if and only if $\mathrm{H}(\mathcal{C})=0$ and $\operatorname{im}(\beta)$ has nonzero finite length.

Proof. Part (a) is immediate from (2.2) in the proof of Proposition 2.1. For (b), suppose $\operatorname{im}(\beta)=0$. Then $\mathrm{H}(\mathcal{C})=Y / \operatorname{im}(\alpha)$. Since $\operatorname{im}(\alpha) \subseteq \mathfrak{m} Y$ and $Y \neq 0$, we cannot have $\mathrm{H}(\mathcal{C})=0$, by Nakayama's lemma.

For (c), suppose first that $P_{\mathfrak{m}}^{\mathcal{C}}(n) \equiv 0$. By (a), we have $\mathrm{H}(\mathcal{C})=0$. From (2.1) and (2.3), we have $\mathfrak{m}^{n} Z \cap \operatorname{im}(\beta)=\mathfrak{m}^{n} \operatorname{im}(\beta)$, for $n$ large. Since $\operatorname{im}(\beta)$ is contained in $\mathfrak{m} Z$, it follows that $\mathfrak{m}^{n-1} \operatorname{im}(\beta)=\mathfrak{m}^{n} \operatorname{im}(\beta)$, so by Nakayama's lemma, $\operatorname{im}(\beta)$ has finite length. By $(b), \operatorname{im}(\beta) \neq 0$. Conversely, suppose that $\mathrm{H}(\mathcal{C})=0$ and $\operatorname{im}(\beta)$ has finite length. Using the notation from the proof of Proposition 2.1, the fact that $U:=\mathrm{H}(\mathcal{C})=0$ implies that $P_{2}(n) \equiv 0$. Since $\operatorname{im}(\beta)$ has finite length, (2.3) and the Artin-Rees lemma imply that $P_{1}(n) \equiv 0$. Since $P_{\mathfrak{m}}^{\mathcal{C}}(n)=P_{1}(n)+P_{2}(n)$, this gives what we want.

Remark 2.4. Regarding Proposition 2.1, note that generally it is the term $\operatorname{dim}(\mathcal{M})-1$ that makes determining the exact degree of $P_{I}^{\mathcal{C}}(n)$ for arbitrary $I$ difficult. Indeed, whenever $\mathrm{H}(\mathcal{C})$ is zero, the degree of $P_{I}^{\mathcal{C}}(n)$ is equal to $\operatorname{dim}(\mathcal{M})-1$. (Think of the case when $I$ is $\mathfrak{m}$-primary and $P_{I}^{\mathcal{C}}(n)=\lambda\left(\operatorname{Tor}_{i}\left(R / I^{n}, R\right)\right)$.) Our success in determining the degree of $P_{\mathfrak{m}}^{\mathcal{C}}(n)$ in Theorem 2.2 and the corresponding results in Section 3 is because, in these cases, we can calculate the dimension of $\mathcal{M}$. Similarly, our success 
in Section 4 with filtrations more general than the $\mathfrak{m}$-adic filtration is due strictly to the ability to calculate the dimension of $\mathcal{M}$ in those cases as well.

\section{§3. The m-adic filtration for the contravariant extension functor}

In this section we apply the results of Section 2 to give a precise formula for the degree of the Hilbert polynomial giving the lengths of the modules $\operatorname{Ext}_{R}^{i}\left(M, N / \mathfrak{m}^{n} N\right)$ and $\operatorname{Tor}_{i}^{R}\left(M, N / \mathfrak{m}^{n} N\right)$. Our formulas below for the degrees of these polynomials (see Theorems 3.2 and 3.12 ) involve the dimension of the image of the $i$ th syzygy of $M$ or its transpose in an appropriate direct sum of copies of $N$ and are immediate consequences of Theorem 2.2. We will show that, in those cases where the dimension of syzygies of $M$ and their transposes are well behaved, our formulas either agree with or improve prior estimates. However, the formulas in Theorems 3.2 and 3.12 are valid in all cases.

We begin by establishing some notation. Let

$$
\mathbf{F}: \quad \cdots \rightarrow F_{i+1} \stackrel{\phi_{i+1}}{\longrightarrow} F_{i} \stackrel{\phi_{i}}{\rightarrow} F_{i-1} \rightarrow \cdots
$$

denote a minimal free resolution of $M$. We set $\beta_{i}(M):=\operatorname{rank}\left(F_{i}\right)$ for all $i$. Thus, for all $i \geq 0, \beta_{i}(M)$ is the $i$ th Betti number of $M$. We can calculate $\operatorname{Ext}^{i}\left(M, N / I^{n} N\right)$ by applying $\operatorname{Hom}\left(-, N / I^{n} N\right)$ to $\mathbf{F}$. Thus, $\operatorname{Ext}^{i}(M, N /$ $\left.I^{n} N\right)$ is the cohomology of the cochain complex $\operatorname{Hom}\left(\mathbf{F}, N / I^{n} N\right)$.

Alternately, we may first apply the functor $\operatorname{Hom}(-, N)$ to the resolution $\mathbf{F}$ to obtain the complex

$$
\operatorname{Hom}(\mathbf{F}, N): \quad \cdots \rightarrow N^{\beta_{i-1}(M)} \stackrel{\overline{\phi_{i}}}{\rightarrow} N^{\beta_{i}(M) \stackrel{\overline{\phi_{i+1}}}{\rightarrow}} N^{\beta_{i+1}(M)} \rightarrow \cdots,
$$

which we then tensor with $R / I^{n}$. The resulting complex $\operatorname{Hom}_{R}(\mathbf{F}, N) \otimes$ $R / I^{n}$ is isomorphic to $\operatorname{Hom}_{R}\left(\mathbf{F}, N / I^{n} N\right)$. Hence we may calculate $\operatorname{Ext}_{R}^{i}(M$, $\left.N / I^{n} N\right)$ as the $i$ th cohomology of $\operatorname{Hom}_{R}(\mathbf{F}, N) \otimes R / I^{n}$. Homology and cohomology of complexes of this form were studied in [7].

\section{Hilbert polynomials for derived functors}

For fixed $i \geq 1$, assume that the modules $\operatorname{Ext}^{i}\left(M, N / I^{n} N\right)$ have finite length for $n$ large. It follows from [7, Corollary 4] that the lengths $\lambda\left(\operatorname{Ext}_{R}^{i}\left(M, N / I^{n} N\right)\right)$ are given by a rational polynomial for $n$ sufficiently large. We will write $\mathcal{E}_{M, N, I}^{i}(n)$ for this polynomial. By [7, Corollary 4], we have the following estimate for the degree of $\mathcal{E}_{M, N, I}^{i}(n)$ :

$$
\operatorname{deg}\left(\mathcal{E}_{M, N, I}^{i}(n)\right) \leq \max \left\{\operatorname{dim}\left(\operatorname{Ext}_{R}^{i}(M, N)\right), \ell_{N}(I)-1\right\}
$$


where $\ell_{N}(I)$ denote the analytic spread of $I$ on $N$. Recall that $\ell_{N}(I)$ is the Krull dimension of the graded module $\bigoplus_{n \geq 0} I^{n} N / \mathfrak{m} I^{n} N$. Equivalently, if we write $S$ for the ring $R / \operatorname{ann}(N)$ and $\mathcal{R}(I S)$ for the Rees ring of $S$ with respect to $I S$, then $\ell_{N}(I)$ is the dimension of the ring $\mathcal{R}(I S) / \mathfrak{m} \mathcal{R}(I S)$; that is, it is just the analytic spread of the image of $I$ in the ring $S$. (See [7, proof of Proposition 3] for a proof of this fact.) Moreover, in [7] it is shown that equality holds in the degree estimate when the first term on the right is at least as large as the second term on the right. Similarly, in [7], it is shown that when, for $i \geq 1$ fixed, the modules $\operatorname{Tor}_{i}^{R}\left(M, N / I^{n} N\right)$ have finite length, those lengths are given by a rational polynomial in $n$, for $n$ sufficiently large. We will write $\tau_{i}^{M, N, I}(n)$ for the corresponding polynomial. In [7], it is shown that

$$
\operatorname{deg}\left(\tau_{i}^{M, N, I}(n)\right) \leq \max \left\{\operatorname{dim}\left(\operatorname{Tor}_{i}(M, N)\right), \ell_{N}(I)-1\right\},
$$

and equality holds when the first term on the right-hand side of the inequality is at least as large as the second term on the right-hand side.

REMARK 3.1. Before stating one of the main results of this section, we first point out that the degree bounds above can be recovered from Proposition 2.1. For the degree of $\mathcal{E}_{M, N, I}^{i}(n)$, it follows from Proposition 2.1 that

$$
\operatorname{deg}\left(\mathcal{E}_{M, N, I}^{i}(n)\right)=\max \left\{\operatorname{dim}\left(\operatorname{Ext}_{R}^{i}(M, N)\right), \operatorname{dim}\left(\mathcal{T}_{i}\right)-1\right\}
$$

for $\mathcal{T}_{i}=\bigoplus_{n \geq 0}\left(I^{n} N^{\beta_{i+1}(M)} \cap \operatorname{im}\left(\overline{\phi_{i+1}}\right)\right) / I^{n} \operatorname{im}\left(\overline{\phi_{i+1}}\right)$. Note that we can regard $\mathcal{T}_{i}$ as a module over the Rees $\operatorname{ring} \mathcal{R}(I S)$ of $S:=R / \operatorname{ann}(N)$ with respect to $I S$. Since, by assumption, the lengths of the graded components of $\mathcal{T}_{i}$ are finite (see the proof of Proposition 2.1), $\mathcal{T}_{i}$ must be annihilated by some power of $\mathfrak{m} \mathcal{R}(I S)$, say, $\mathfrak{m}^{q} \mathcal{R}(I S)$. Then $\operatorname{dim}\left(\mathcal{T}_{i}\right) \leq \operatorname{dim}\left(\mathcal{R}(I S) / \mathfrak{m}^{q} \mathcal{R}(I S)\right)=$ $\ell_{N}(I)$, which gives what we want. The argument for the degree bound involv$\operatorname{ing} \tau_{i}^{M, N, I}(n)$ is entirely analogous.

We are now ready for the main result of this section.

TheOREM 3.2. Let $(R, \mathfrak{m})$ be a local ring, and let $M$ and $N$ be finitely generated $R$-modules. Fix $0 \leq i \leq$ p.d. $(M)$. Set $T_{i}:=\operatorname{im}\left(\overline{\phi_{i+1}}\right)$, for $\overline{\phi_{i+1}}$ as above. Then

$$
\operatorname{deg}\left(\mathcal{E}_{M, N, \mathfrak{m}}^{i}(n)\right)=\max \left\{\operatorname{dim}\left(\operatorname{Ext}^{i}(M, N)\right), \operatorname{dim}\left(T_{i}\right)-1\right\} .
$$

Proof. If we use the fact above that $\operatorname{Ext}_{R}^{i}\left(M, N / \mathfrak{m}^{n} N\right)$ is the $i$ th cohomology of the complex $\operatorname{Hom}(\mathbf{F}, N) \otimes R / \mathfrak{m}^{n}$, then the theorem follows immediately from Theorem 2.2. 
REMARK 3.3. (i) Since the resolution $\mathbf{F}$ is minimal, it follows from Corollary 2.3 that $\mathcal{E}_{M, N, \mathfrak{m}}^{i}(n)$ is identically zero if and only if $\operatorname{Ext}^{i}(M, N)=0$ and $T_{i}$ has nonzero finite length. Therefore, if we adopt the conventions that the zero polynomial has degree -1 and the zero module has dimension $-\infty$, Theorem 3.2 does give the correct value for the degree of $\mathcal{E}_{M, N, \mathfrak{m}}^{i}(n)$ in this case.

(ii) Concerning the upper bound for the degree of $\mathcal{E}_{M, N, I}^{i}(n)$ given before Remark 3.1, when $I$ is an $\mathfrak{m}$-primary ideal, in particular, when $I=\mathfrak{m}$, $\ell_{N}(I)=\operatorname{dim}(N)$. Thus, since $\operatorname{dim}\left(T_{i}\right) \leq \operatorname{dim}(N)$, we see that Theorem 3.2 improves the estimate from [7] in the special case that $\mathfrak{m}=I$. This improvement is extended to more general filtrations (but not all modules) in Section 4 .

(iii) The Hilbert polynomial $\tau_{i}^{M, R, \mathfrak{m}}(n)$ giving the lengths of $\operatorname{Tor}_{i}(M, R /$ $\mathfrak{m}^{n}$ ) has degree less than $d$ for all $i>0$ (see [5] or Theorem 3.12 below). This no longer holds for $\mathcal{E}_{M, R, \mathfrak{m}}^{i}(n)$. Indeed, let $R$ be a local ring with a prime ideal $P$ of maximal dimension such that $R_{P}$ is not Gorenstein, and set $M:=R / P$. Then $\operatorname{Ext}^{i}(M, R)_{P} \neq 0$ for all $i>0$, so that $\operatorname{dim}\left(\operatorname{Ext}^{i}(M, R)\right)=d$, for all $i>0$. Thus, by Theorem $3.2, \operatorname{deg}\left(\mathcal{E}_{M, R, \mathfrak{m}}^{i}(n)\right)=d$, for all $i>0$.

Corollary 3.4. Suppose that $M$ has finite p.d., say, p.d. $(M)=i$. Then $\operatorname{deg}\left(\mathcal{E}_{M, N, \mathfrak{m}}^{i}(n)\right)=\operatorname{dim}\left(\operatorname{Ext}^{i}(M, N)\right)$.

Proof. This is immediate from the theorem, since in this case, $T_{i}=0$.

In Theorem 3.2, we may replace $T_{i}$ by $N$ in any number of situations, as the following corollary shows.

Corollary 3.5. Let $(R, \mathfrak{m})$ be a local ring, and let $M$ and $N$ be finitely generated $R$-modules. Fix $0 \leq i<$ p.d. $(M)$, and suppose that one of the following conditions holds:

(a) $i=0$;

(b) there exists a prime ideal $P$ of maximal dimension in the support of $N$ so that some entry of $\phi_{i+1}($ say, $r)$ does not belong to $P$ (e.g., $r$ is a nonzero divisor on $N)$;

(c) $\beta_{i}(M)>\beta_{i-1}(M)$;

(d) $N=R$, and $M_{P}$ is not a free $R_{P}$-module, for some prime ideal $P \subseteq R$ of dimension $d$.

Then

$$
\operatorname{deg}\left(\mathcal{E}_{M, N, \mathfrak{m}}^{i}(n)\right)=\max \left\{\operatorname{dim}\left(\operatorname{Ext}^{i}(M, N)\right), \operatorname{dim}(N)-1\right\}
$$


Proof. For (a), we have an exact sequence

$$
0 \rightarrow \operatorname{Hom}(M, N) \rightarrow N^{\beta_{0}} \stackrel{\overline{\phi_{1}}}{\rightarrow} T_{0} \rightarrow 0
$$

By Theorem 3.2, if $\operatorname{dim}(\operatorname{Hom}(M, N))=\operatorname{dim}(N), \operatorname{deg}\left(\mathcal{E}_{M, N, I}^{0}(n)\right)=\operatorname{dim}(N)$, which is the maximum value in question. Otherwise, the exact sequence above shows that $T_{0}$ and $N$ have the same dimension, so $\operatorname{dim}\left(T_{0}\right)-1=$ $\operatorname{dim}(N)-1$ is the maximum value, and this gives what we want.

For (b), since $r_{P} \cdot N_{P} \neq 0,\left(T_{i}\right)_{P}=\operatorname{im}\left(\overline{\phi_{i+1}}\right)_{P} \neq 0$. Thus, $\operatorname{dim}\left(T_{i}\right)=$ $\operatorname{dim}(N)$, and this gives what we want by Theorem 3.2.

To prove (c), let $P$ be a prime ideal of maximal dimension in the support of $N$. By Theorem 3.2, it suffices to show that either $\operatorname{Ext}^{i}(M, N)_{P} \neq 0$ or $\left(T_{i}\right)_{P} \neq 0$. If $\operatorname{Ext}^{i}(M, N)_{P}=0$ and $\left(T_{i}\right)_{P}=0$, then $\left(\overline{\phi_{i}}\right)_{P}$ is surjective. But this cannot happen if $\beta_{i}(M)>\beta_{i-1}(M)$.

For $(\mathrm{d})$, by assumption, $\left(\phi_{i+1}\right)_{P} \neq 0$, so $\left(T_{i}\right)_{P} \neq 0$, which gives the result.

It is clear that as long as any one of the conditions (a)-(d) of Corollary 3.5 is met, we obtain $\operatorname{deg}\left(\mathcal{E}_{M, N, \mathfrak{m}}^{i}(n)\right)=\operatorname{dim}(N)-1$ whenever the dimension of $\operatorname{Ext}^{i}(M, N)$ is less than $\operatorname{dim}(N)$. We list a couple of such cases in the following corollary.

Corollary 3.6. Let $(R, \mathfrak{m})$ be a local ring, and let $M$ and $N$ be finitely generated $R$-modules. Fix $0 \leq i<$ p.d. $(M)$, and suppose that one of the following conditions holds.

(a) $M$ has a rank, and $\operatorname{dim}(N)=\operatorname{dim}(R)$.

(b) $M$ is a nonzero syzygy of $\mathrm{k}$, and $\operatorname{dim}(N) \geq 1$.

Then $\operatorname{deg}\left(\mathcal{E}_{M, N, \mathfrak{m}}^{i}(n)\right)=\operatorname{dim}(N)-1$.

Proof. Let $P$ be a prime of maximal dimension in the support of $N$. In (a), $P$ is a minimal prime of $R$. Therefore, by [5, Remark 2.1], in either (a) or $(\mathrm{b})$, the image of $\left(\phi_{i+1}\right)_{P}$ is a nonzero summand of $\left(F_{i}\right)_{P}$. Thus, in each case, at least one entry of $\left(\phi_{i+1}\right)_{P}$ is a unit. Therefore, in both (a) and (b), the conclusion of Corollary 3.5 holds; that is,

$$
\operatorname{deg}\left(\mathcal{E}_{M, N, \mathfrak{m}}^{i}(n)\right)=\max \left\{\operatorname{dim}\left(\operatorname{Ext}^{i}(M, N)\right), \operatorname{dim}(N)-1\right\} .
$$

On the other hand, if (a) holds, it follows that $\operatorname{dim}\left(\operatorname{Ext}^{i}(M, N)\right)<d=$ $\operatorname{dim}(N)$, so the maximum value in question is $\operatorname{dim}(N)-1$. Similarly, if (b) holds, then $\operatorname{dim}\left(\operatorname{Ext}^{i}(M, N)\right)=0$, so again, the maximum in question is $\operatorname{dim}(N)-1$, which completes the proof. 
Corollary 3.7. If $\beta_{i}(M)=\beta_{i-1}(M)$, then $\operatorname{deg}\left(\mathcal{E}_{M, N, \mathfrak{m}}^{i}(n)\right) \geq \operatorname{dim}(N)-$ 2.

Proof. By dimension shifting, we may assume $i=1$. Then we have an exact sequence

$$
0 \rightarrow \frac{\mathfrak{m}^{n} N}{\mathfrak{m}^{n+1} N} \rightarrow \frac{N}{\mathfrak{m}^{n+1} N} \rightarrow \frac{N}{\mathfrak{m}^{n} N} \rightarrow 0
$$

from which we obtain

$$
\begin{aligned}
0 & \rightarrow \operatorname{Hom}\left(M, \mathfrak{m}^{n} N / \mathfrak{m}^{n+1} N\right) \rightarrow \operatorname{Hom}\left(M, N / \mathfrak{m}^{n+1} N\right) \\
& \rightarrow \operatorname{Hom}\left(M, N / \mathfrak{m}^{n} N\right) \rightarrow \operatorname{Ext}^{1}\left(M, \mathfrak{m}^{n} N / \mathfrak{m}^{n+1} N\right) \\
& \stackrel{\rho_{n}}{\rightarrow} \operatorname{Ext}^{1}\left(M, N / \mathfrak{m}^{n+1} N\right)
\end{aligned}
$$

Since the resolution of $M$ is minimal, the lengths of

$$
\operatorname{Hom}\left(M, \mathfrak{m}^{n} N / \mathfrak{m}^{n+1} N\right), \quad \operatorname{Ext}^{1}\left(M, \mathfrak{m}^{n} N / \mathfrak{m}^{n+1} N\right)
$$

are just $\beta_{0}(M) \cdot L(n)$ and $\beta_{1}(M) \cdot L(n)$, respectively, where $L(n)$ is the polynomial giving the minimal number of generators of $\mathfrak{m}^{n} N$ for $n$ large. Using the fact that $\beta_{1}(M)=\beta_{0}(M)$, it follows that

$$
\mathcal{E}_{M, N, \mathfrak{m}}^{1}(n+1) \geq \mathcal{E}_{M, N, \mathfrak{m}}^{0}(n+1)-\mathcal{E}_{M, N, \mathfrak{m}}^{0}(n) .
$$

By Corollary $3.5, \mathcal{E}_{M, N, \mathfrak{m}}^{0}(n)$ has degree greater than or equal to $\operatorname{dim}(N)-1$, and this gives what we want.

REMARK 3.8. Let $R$ be a two-dimensional local ring of depth one, and suppose that $a \in R$ is a parameter such that $(0:(0: a))=(a)$. Then for $M:=R /(a)$ and $i=1$, we have that $\mathcal{E}_{M, R, \mathfrak{m}}^{1}(n)$ is a nonzero constant. In particular, this gives an example where $\beta_{0}(M)=\beta_{1}(M)$ and $\operatorname{deg}\left(\mathcal{E}_{M, R, \mathfrak{m}}^{1}(n)\right)=$ $\operatorname{dim}(R)-2$, that is, an example where the lower bound in Corollary 3.7 is attained. To see this, let

$$
\cdots \rightarrow R^{r} \stackrel{\phi_{2}}{\rightarrow} R \stackrel{\cdot a}{\rightarrow} R \rightarrow M \rightarrow 0
$$

be the start of a minimal resolution of $M$. Note that the image of $\phi_{2}$ is just $(0: a)$. To calculate $\operatorname{Ext}^{1}(M, R)$, we look at the dual of the resolution, thereby getting the complex

$$
0 \rightarrow R \stackrel{\cdot a}{\rightarrow} R \stackrel{\phi_{2}^{t}}{\rightarrow} R^{r} \rightarrow \cdots
$$


Note that $\operatorname{ker}\left(\phi_{2}^{t}\right)=(0:(0: a))$, so by our assumption, $\operatorname{Ext}^{1}(M, R)=0$. On the other hand, clearly $a$ belongs to the annihilator of $T:=\operatorname{im}\left(\phi_{2}^{t}\right)$, so $\operatorname{dim}(T) \leq 1$. Since the depth of $R$ is one, $T$ cannot be zero-dimensional. Thus, $\operatorname{dim}(T)=1$, so by Theorem $3.2, \mathcal{E}_{M, R, \mathfrak{m}}^{1}(n)$ is a nonzero constant.

To find a concrete example with the stated properties, it suffices to find a one-dimensional local ring $(S, \mathfrak{n})$ with a parameter $a$ satisfying $(0:(0$ : $a S))=(a)$. Indeed, given such an $S$ and $a$, let $R:=S[X]_{(\mathfrak{n}, X)}$, where $X$ is an indeterminate over $S$. Then $R$ and $a$ meet the requirements stated above. Finally, to find such an $S$, we use the following example shown to us by Craig Huneke.

EXAMPLE 3.9. Let $\mathrm{k}$ be a field, and let $x, y, z, u$, and $v$ be indeterminates. Let $S$ denote the power series ring $\mathrm{k}[[x, y, z, u, v]]$ modulo the ideal $I$, where $I$ is the ideal generated by $x^{2}, x z, z^{2}, x u, z v, u^{2}, v^{2}, z u+x v+u v, y u, y v, y x-$ $z u, y z-x v$. Then $S$ is a one-dimensional local ring with parameter ideal $y S$ satisfying $(0:(0: y S))=y S$.

For a deeper analysis of this situation, the interested reader should consult $[4]$.

Corollaries 3.6 and 3.7 together with Theorem 3.2 yield some information on the dimension of syzygies over an arbitrary local ring. In Section 5, we record some of the consequences that our work has for the dimension of syzygies.

In our last corollary of this section concerning the contravariant extension functor, we record what happens when $N=R$ is a Cohen-Macaulay local ring with a canonical module.

Corollary 3.10. Suppose that $R$ is a Cohen-Macaulay local ring with canonical module $\omega$. Assume that $0 \leq i<$ p.d.( $\omega)$. Then,

(a) $\operatorname{deg}\left(\mathcal{E}_{\omega, R, \mathfrak{m}}^{i}(n)\right)=\max \left\{\operatorname{dim}\left(\operatorname{Ext}^{i}(\omega, R)\right), \operatorname{dim}(R)-1\right\}$, and

(b) $\operatorname{deg}\left(\mathcal{E}_{\omega, R, \mathfrak{m}}^{i}(n)\right)=\operatorname{dim}(R)-1$, if $R$ is generically Gorenstein.

Moreover, if $i=0$, then $\operatorname{deg}\left(\mathcal{E}_{\omega, R, \mathfrak{m}}^{0}(n)\right)=\operatorname{dim}(R)$.

Proof. We consider (a) and (b) together. Suppose there exists a minimal prime $P \subseteq R$ such that $R_{P}$ is not Gorenstein. Then $\omega_{P}$ is not a free $R$-module, so (a) holds by Corollary 3.5(d). Otherwise, $R$ is generically Gorenstein, so (b) holds by Corollary 3.6(a).

Now suppose that $i=0$, and let $P \subseteq R$ be a prime of maximal dimension. Then $\operatorname{Hom}(\omega, R)_{P} \neq 0$, so $\operatorname{dim}(\operatorname{Hom}(\omega, R))=d$, and the result follows from (a). 
REMARK 3.11. Returning to the setup at the beginning of this section, let $\tau_{i}^{M, N, \mathfrak{m}}(n)$ denote the polynomial giving the lengths of $\operatorname{Tor}_{i}\left(M, N / \mathfrak{m}^{n} N\right)$, for $n$ large. Then $\operatorname{Tor}_{i}\left(M, N / \mathfrak{m}^{n} N\right)$ is the $i$ th homology in the complex $(\mathcal{F} \otimes N) \otimes R / \mathfrak{m}^{n}$, so Theorem 2.2 yields a formula for the degree of $\tau_{i}^{M, N, \mathfrak{m}}(n)$ analogous to the one obtained in Theorem 3.2 for $\mathcal{E}_{M, N, \mathfrak{m}}^{i}(n)$. When $N=R$, several special cases were given in [5]. Note that one can then list several corollaries to Theorem 3.12 analogous to those above.

TheOREM 3.12. Let $(R, \mathfrak{m})$ be a local ring, and let $M$ and $N$ be finite $R$-modules. Fix $0 \leq i \leq$ p.d. $(M)$, and let $\tau_{i}^{M, N, \mathfrak{m}}(n)$ denote the Hilbert polynomial giving the lengths of the modules $\operatorname{Tor}_{i}\left(M, N / \mathfrak{m}^{n} N\right)$ for $n$ large. Let $C_{i}$ denote the image of the induced map $\overline{\phi_{i} \otimes 1_{N}}: F_{i} \otimes N \rightarrow F_{i-1} \otimes N$. Then

$$
\operatorname{deg}\left(\tau_{i}^{M, N, \mathfrak{m}}(n)\right)=\max \left\{\operatorname{dim}\left(\operatorname{Tor}_{i}(M, N)\right), \operatorname{dim}\left(C_{i}\right)-1\right\}
$$

In particular, if $N=R$ and $i \geq 1$, then

$$
\operatorname{deg}\left(\tau_{i}^{M, N, \mathfrak{m}}(n)\right)=\operatorname{dim}\left(\Omega_{R}^{i}(M)\right)-1
$$

Proof. For the first statement, we just apply Theorem 2.2 to the $i$ th spot of the complex $\mathbf{F} \otimes N$, where, as before, $\mathbf{F}$ denotes the minimal resolution of $M$. The second statement follows immediately from the first.

REMARK 3.13. (i) Assume that $N=R$ and $i \geq 1$. Then the theorem above shows that the degree of $\tau_{i}^{M, N, \mathfrak{m}}(n)$ is simply the dimension of the $i$ th syzygy of $M$ minus one. On the other hand, for $N=R$ and $i \geq 1$, the degree of $\mathcal{E}_{M, N, \mathfrak{m}}^{i}(n)$ is determined by both the dimension of the module $\operatorname{Ext}^{i}(M, R)$ and the dimension of $T_{i}$, which in this case is the dimension of the $(i+1)$ st syzygy of $M$. Suppose that $M$ and $R$ are such that $\operatorname{Ext}^{i}(M, R)$ has dimension less than or equal to $d-1$ (e.g., $R$ is generically Gorenstein or $M$ has a rank). Then the degree of $\mathcal{E}_{M, R, \mathfrak{m}}^{i}(n)$ is less than or equal to $d-1$. Now, since either the $i$ th or the $(i+1)$ st syzygy of $M$ must have dimension equal to the dimension of $R$, it follows that either $\tau_{i}^{M, R, \mathfrak{m}}(n)$ or $\mathcal{E}_{M, R, \mathfrak{m}}^{i}(n)$ has maximal degree $\operatorname{dim}(R)-1$. Of course, as above, very minor assumptions on $M$ will also guarantee that both polynomials have maximal degree. However, as pointed out in Question 5.1, for $R$ and $M$ arbitrary, it is not known whether the dimensions of the syzygies of $M$ ultimately stabilize, so one cannot make a definitive statement regarding the degrees of $\mathcal{E}_{M, R, \mathfrak{m}}^{i}(n)$ and $\tau_{i}^{M, R, \mathfrak{m}}(n)$, even for $i$ sufficiently large. 
(ii) In [3], it is shown that for $i>0$,

$$
\operatorname{depth}(R)-1 \leq \operatorname{deg}\left(\tau_{i}^{M, R, \mathfrak{m}}(n)\right) \leq \operatorname{dim}(R)-1 .
$$

By Theorem 3.12, we have $\operatorname{deg}\left(\tau_{i}^{M, R, \mathfrak{m}}(n)\right)=\operatorname{dim}\left(\Omega_{R}^{i}(M)\right)-1$. Now on the one hand, $\operatorname{depth}(R)=\operatorname{depth}\left(F_{i}\right) \leq \operatorname{dim}\left(\Omega_{R}^{i}(M)\right)$, while on the other hand, $\operatorname{dim}\left(\Omega_{R}^{i}(M)\right) \leq \operatorname{dim}(R)$, so our result improves the upper and lower bounds for the degree of $\tau_{i}^{M, R, \mathfrak{m}}(n)$ given in [3]. Furthermore, if $M$ has a rank and $N=R$, it is shown in [5] that $\operatorname{deg}\left(\tau_{i}^{M, R, \mathfrak{m}}(n)\right)=\operatorname{dim}(R)-1$. Since in this case, $R, M$, and $\Omega_{R}^{i}(M)$ all have the same dimension, Theorem 3.12 recovers this result as well.

\section{$\S 4$. More general filtrations}

We now turn to giving an analogue of the main results in [5] for $\operatorname{Ext}^{i}(M$, $\left.N / I^{n} N\right)$. In [5], the second and fourth authors considered the Hilbert polynomial giving the lengths of $\operatorname{Tor}_{i}\left(M, R / I^{n}\right)$. In that paper, various assumptions were made on $I$ and $M$ which forced $\tau_{i}^{M, N, I}(n)$ to have maximal degree $\ell(I)-1$. Roughly speaking, the assumptions on $M$ were made so that the $i$ th syzygy has maximal dimension. The assumptions on the filtrations given in [5] were made in order to replicate some of the properties satisfied by the $\mathfrak{m}$-adic filtration. The reason for this is now clear in light of Theorem 3.12. Likewise, we may use some of the ideas underlying Theorem 3.2 to give the corresponding results for $\mathcal{E}_{M, N, I}^{i}(n)$ for similar $I$ and $M$.

Before presenting our main results, we state a proposition which is simply a restatement of Proposition 2.1 in the context of the contravariant extension functor. For the sake of consistent notation with Section 3, we set $T_{i}:=\operatorname{im}\left(\overline{\phi_{i+1}}\right)$, and we set

$$
\mathcal{T}_{i}:=\bigoplus_{n \geq 0}\left(I^{n} N^{\beta_{i+1}(M)} \cap T_{i}\right) / I^{n} T_{i} .
$$

Note that, in our present context, $\mathcal{T}_{i}$ is just $\mathcal{M}$ from Proposition 2.1.

Proposition 4.1. Let $(R, \mathfrak{m})$ be a local ring, and suppose that $M$ and $N$ are finitely generated $R$-modules. Fix $0 \leq i \leq$ p.d. $(M)$, and let $I \subseteq R$ be an ideal. Assume that, for all large $n$, the lengths of $\operatorname{Ext}^{i}\left(M, N / I^{n} N\right)$ are nonzero and finite. Then

$$
\operatorname{deg}\left(\mathcal{E}_{M, N, I}^{i}(n)\right)=\max \left\{\operatorname{dim}\left(\operatorname{Ext}^{i}(M, N)\right), \operatorname{dim}\left(\mathcal{T}_{i}\right)-1\right\}
$$


We now give an analogue of Theorem 3.2 for ideals divisible by $\mathfrak{m}$.

THEOREM 4.2. Let $(R, \mathfrak{m})$ be a local ring, and suppose that $M$ and $N$ are finitely generated $R$-modules. Let $I$ be an ideal divisible by $\mathfrak{m}$, that is, $I=\mathfrak{m} C$, for some ideal $C \subseteq R$. Fix $0 \leq i \leq$ p.d.(M), and suppose that the lengths of $\operatorname{Ext}^{i}\left(M, N / I^{n} N\right)$ are nonzero and finite for $n$ large. Then,

$$
\operatorname{deg}\left(\mathcal{E}_{M, N, I}^{i}(n)\right)=\max \left\{\operatorname{dim}\left(\operatorname{Ext}^{i}(M, N)\right), \ell_{T_{i}}(I)-1\right\},
$$

where as before, $T_{i}:=\operatorname{im}\left(\overline{\phi_{i+1}}\right)$.

Proof. By Proposition 4.1, it suffices to prove $\operatorname{dim}\left(\mathcal{T}_{i}\right)=\ell_{T_{i}}(I)$. Consider the filtration $\mathcal{J}$ whose terms are $J_{n}:=\mathfrak{m}^{n-1} C^{n}, n \geq 1$. Note that $J_{n+1}=I J_{n}$ for all $n \geq 1$. Then $\mathcal{J}$ is an $I$-good filtration, so by [5, Proposition 2.2], the graded module $\bigoplus_{n \geq 0} J_{n} T_{i} / \mathfrak{m} J_{n} T_{i}$ has dimension $\ell_{T_{i}}(I)$. Now, on the one hand, since $\mathbf{F}$ is a minimal resolution, $J_{n} T_{i} \subseteq I^{n} N^{\beta_{i+1}(M)} \cap T_{i}$. Thus,

$$
\bigoplus_{n \geq 0} J_{n} T_{i} / \mathfrak{m} J_{n} T_{i} \subseteq \mathcal{T}_{i}
$$

from which it follows that $\mathcal{T}_{i}$ has dimension at least $\ell_{T_{i}}(I)$. On the other hand, set $S:=R / \operatorname{ann}\left(T_{i}\right)$. Then $\mathcal{T}_{i}$ is a finitely generated module over the Rees algebra $\mathcal{R}(I S)$ of $S$ with respect to $I S$ whose graded components have finite length. Thus, there exists $r>0$ such that $\mathfrak{m}^{r} \mathcal{R}(I S)$ annihilates $\mathcal{T}_{i}$. Therefore,

$$
\operatorname{dim}\left(\mathcal{T}_{i}\right) \leq \operatorname{dim}\left(\mathcal{R}(I S) / \mathfrak{m}^{r} \mathcal{R}(I S)\right)=\ell_{S}(I)=\ell_{T_{i}}(I)
$$

Thus, $\operatorname{dim}\left(\mathcal{T}_{i}\right)=\ell_{T_{i}}(I)$, which gives what we want.

REMARK 4.3. For $I=\mathfrak{m} C$ and $I T_{i} \neq 0$, the value of $\ell_{T_{i}}(I)$ can vary anywhere between zero and $\operatorname{dim}\left(T_{i}\right)$, and the latter can be as large as $\operatorname{dim}(R)$. However, if we set $S:=R / \operatorname{ann}\left(T_{i}\right)$ and assume that height $(I S)>0$, then $\ell_{T_{i}}(I)$ achieves its maximum value of $\operatorname{dim}\left(T_{i}\right)$. To see this, after a change in notation, it suffices to see that if height $(I)>0$, then $\ell(I)=d=\operatorname{dim}(R)$. For this, recall that since height $(C)>0$, the Hilbert-Samuel polynomial giving the lengths of the modules $C^{r} \mathfrak{m}^{s} / C^{r} \mathfrak{m}^{s+1}$ for $r, s$ large is a polynomial of total degree $d-1$ in $r$ and $s$ with nonnegative leading coefficients. For large $n$, we set $n:=r=s$. It follows that the polynomial giving the lengths of $(C \mathfrak{m})^{n} / \mathfrak{m}(C \mathfrak{m})^{n}=I^{n} / \mathfrak{m} I^{n}$ has degree $d-1$. Therefore, $\ell(I)=d$.

If $N=R$, we have an immediate corollary for ideals divisible by $\mathfrak{m}$. 
Corollary 4.4. Let $R$ be a local ring, and let $M$ be a finitely generated $R$-module. Assume that $R$ is unmixed and equidimensional. Assume further that either $M$ has a rank or $R$ is generically Gorenstein. Let $I=\mathfrak{m} C$ be an ideal of $R$ such that height $(I)>0$. Then, $\operatorname{deg}\left(\mathcal{E}_{M, R, I}^{i}(n)\right)=d-1$.

Proof. If $M$ has a rank or $R$ is generically Gorenstein, $\operatorname{dim}\left(\operatorname{Ext}^{i}(M, R)\right) \leq$ $d-1$. Thus, by Theorem 4.2 and its proof, it suffices to show that $\ell_{T_{i}}(I)=d$. Consider the $(i+1)$ st syzygy of $M, K_{i}:=\operatorname{im}\left(\phi_{i+1}\right)$. Since $K_{i}$ and $T_{i}$ have the same support, we just have to show that $\ell_{K_{i}}(I)=d$. Let $P$ be any prime minimal in the support of $K_{i}$. Since $R$ is unmixed and equidimensional, $S:=R / P$ has dimension $d$. Since height $(I)>0$, height $(I S)>0$. By the remark above, $\ell_{S}(I)=d$. Since $\ell_{K_{i}}(I)$ is the maximum value over all such $S$, it follows that $\ell_{K_{i}}(I)=d$, which is what we want.

Our final goal is to state a theorem that is a variant for the contravariant extension functor of the main results in [5]. It gives a number of cases where the degree of $\mathcal{E}_{M, N, I}^{i}(n)$ is $d-1$. First, we require a definition and a lemma. In the lemma, we maintain the notation established throughout this paper.

Definition 4.5. Let $M$ be a finitely generated $R$-module; $M$ is said to test finite projective dimension if, for all finitely generated modules $N, N$ has finite projective dimension if and only if for some $i>0, \operatorname{Tor}_{i}^{R}(M, N)=0$.

While the residue field $k$ obviously satisfies this condition-and this is the case one is often interested in - it follows from [1, Corollary 3.3] that $R / J$ satisfies the condition for any integrally closed $\mathfrak{m}$-primary ideal $J$. Of course, if $M$ tests finite projective dimension, then so does any syzygy of $M$.

LEMMA 4.6. Let $N$ be a finitely generated $R$-module such that $N_{P} \neq 0$, for every minimal prime $P$. Let $M$ be a finitely generated $R$-module such that either $M$ has a rank or $M$ is free of constant rank on the punctured spectrum of $R$ and $M$ tests finite projective dimension. Assume further that $i<$ p.d. $(M)$. Then for $\overline{\phi_{i+1}}$ as in Section 3 and $T_{i}:=\operatorname{im}\left(\overline{\phi_{i+1}}\right)$, the annihilator of $T_{i}$ is nilpotent.

Proof. Set $K_{i}:=\operatorname{im}\left(\phi_{i+1}\right)$. Then $\left(K_{i}\right)_{P} \neq 0$ for all minimal primes $P \subseteq R$. When $M$ has a rank, this follows from [5, Remark 2.1]. If $M$ is free of constant rank on the punctured spectrum of $R$ and $M$ tests finite projective dimension, this was shown for finite length $M$ in [5, proof of Theorem 3.3, first paragraph], but for the reader's convenience, we repeat the argument in this slightly more general case. First, note that if $\operatorname{depth}(R)>0$, then $M$ 
has a rank, and we are in the previous case. Suppose that $\operatorname{depth}(R)=0$. By hypothesis, $K_{i}$ is also free of constant rank on the punctured spectrum of $R$. If this locally constant rank were zero, then $K_{i}$ would have finite length. But then by Corollary 2.3(c), $\operatorname{Tor}_{i}\left(R / \mathfrak{m}^{n}, M\right)=0$ for large $n$. By the hypothesis on $M, R / \mathfrak{m}^{n}$ has finite projective dimension, which cannot be when $\operatorname{depth}(R)=0$. Thus $K_{i}$ does not have finite length and thus must be nonzero when localized at any nonmaximal prime ideal. In particular, $\left(K_{i}\right)_{P}$ is nonzero for each minimal prime $P$. Thus, in both cases, the map $\left(\phi_{i+1}\right)_{P}$ is nonzero for each such $P$, and so its transpose $\left(\phi_{i+1}^{t}\right)_{P}$ is also nonzero. Since the $\mathbf{F}_{P}$ is split exact for all minimal primes $P$, the complex $\operatorname{Hom}(\mathbf{F}, N)_{P}$ is also split exact, and since $N_{P}$ is nonzero for each minimal prime $P$, it follows that $\left(T_{i}\right)_{P}$ is not zero for each minimal prime $P$. Thus, the annihilator of $T_{i}$ is nilpotent.

THEOREM 4.7. Let $(R, \mathfrak{m})$ be a local ring of dimension $d$, and let $I \subseteq R$ be an ideal having analytic spread $d$. Let $N$ and $M$ be finitely generated $R$-modules such that $\lambda\left(\operatorname{Ext}^{i}\left(M, N / I^{n} N\right)\right)$ is finite for $n$ large. Here, $0<i<$ p.d.( $M)$. Assume that $M$ has a rank (possibly zero) or that $M$ is free of constant rank on the punctured spectrum of $R$ and $M$ tests finite projective dimension. Assume further that $N_{P}$ is nonzero for every minimal prime $P$. Suppose that one of the following conditions is satisfied:

(i) $I=\mathfrak{m} C$ for some ideal $C \subseteq R$;

(ii) $\left(\mathfrak{m} I^{n} N:_{N} \mathfrak{m}\right)=I^{n} N$, for large $n$;

(iii) $\left(I^{n}:_{R} \mathfrak{m}\right) \nsubseteq \overline{I^{n}}$, for some $n$, and $R$ is quasi unmixed.

Then $\operatorname{deg}\left(\mathcal{E}_{M, N, I}^{i}(n)\right)=d-1$.

Proof. We first note that either assumption on $M$ yields $\operatorname{dim}\left(\operatorname{Ext}^{i}(M\right.$, $N)) \leq d-1$. Thus, by Proposition 4.1, we must show that $\operatorname{dim}\left(\mathcal{T}_{i}\right)=d$. The proof of this for each of the stated conditions follows closely the proofs given for [5, Theorems 3.3 and 3.4]. We will try to give a convincing account without repeating all of the details from [5]. A crucial point in each case is that the annihilator of $T_{i}$ is nilpotent, by Lemma 4.6 .

Now suppose that $I=\mathfrak{m} C$, for some ideal $C$. From the proof of Theorem 4.2, we know that $\operatorname{dim}\left(\mathcal{T}_{i}\right)=\ell_{T_{i}}(I)$. By Lemma 4.6, $T_{i}$ has a nilpotent annihilator. Thus $\ell_{T_{i}}(I)=\ell(I)=d$, which gives what we want.

Suppose that $\left(\mathfrak{m} I^{n} N:_{N} \mathfrak{m}\right)=I^{n} N$ for large $n$. Replacing $I$ by $I^{t}$ for $t$ sufficiently large allows us to show, just as in [5, proof of Theorem 3.3, 
paragraph 3], that for all $n$, we have an equality of socles,

$$
\operatorname{Soc}\left(\mathcal{T}_{i}\right)=\operatorname{Soc}\left(I^{n-1} T_{i} / I^{n} T_{i}\right)
$$

Since $T_{i}$ has a nilpotent annihilator, $d=\ell(I)=\ell_{T_{i}}(I)$, and the same proof used in [5, paragraph 4, page 3079] shows that the module $\bigoplus_{n \geq 0} \operatorname{Soc}\left(I^{n-1} T_{i} /\right.$ $I^{n} T_{i}$ ) has dimension $d$. This in turn implies that $\mathcal{T}_{i}$ also has dimension $d$, which is what we want.

Finally, suppose that $\left(I^{n}: \mathfrak{m}\right) \nsubseteq \overline{I^{n}}$ for some $n$, and suppose that $R$ is quasi unmixed. Again, since the resolution $\mathcal{F}$ is a minimal resolution, $\left(I^{n}: \mathfrak{m}\right) T_{i} \subseteq I^{n} N^{u} \cap T_{i}$. Thus,

$$
\bigoplus_{n \geq 0}\left(I^{n}: \mathfrak{m}\right) T_{i} / I^{n} T_{i} \subseteq \mathcal{T}_{i}
$$

Since the annihilator of $T_{i}$ is nilpotent, the same proof used in [5, proof of Theorem 3.4, paragraphs 3 and 4] shows that the module $\bigoplus_{n>0}\left(I^{n}: \mathfrak{m}\right) T_{i} /$ $I^{n} T_{i}$ has dimension $d$. Therefore, $\mathcal{T}_{i}$ also has dimension $d$, and the proof is complete.

REMARK 4.8. One should note that no assumption is made about the nonvanishing of the extension modules $\operatorname{Ext}^{i}\left(M, N / I^{n} N\right)$ in the theorem. Thus, in particular, the theorem shows that, for ideals and modules as in the theorem, $\operatorname{Ext}^{i}\left(M, N / I^{n} N\right)$ is not zero. A similar remark applies to the corresponding torsion modules.

REMARK 4.9. Since $N$ is not injective, one does not automatically obtain the analogue of results for $\operatorname{Ext}^{i+1}\left(M, I^{n} N\right)$ from $\operatorname{Ext}^{i}\left(M, N / I^{n} N\right)$ by dimension shifting. However, for the question we are interested in, if we assume that the modules $\operatorname{Ext}^{i}(M, N), \operatorname{Ext}^{i}\left(M, N / I^{n} N\right)$, and $\operatorname{Ext}^{i+1}\left(M, I^{n} N\right)$ all have finite length for large $n$, the answer follows readily. Indeed, for all $n \geq 1$, the short exact sequence

$$
0 \rightarrow I^{n} N \rightarrow N \rightarrow N / I^{n} N \rightarrow 0
$$

gives rise to the long exact sequence

$$
\operatorname{Ext}^{i}(M, N) \rightarrow \operatorname{Ext}^{i}\left(M, N / I^{n} N\right) \rightarrow \operatorname{Ext}^{i+1}\left(M, I^{n} N\right) \rightarrow \operatorname{Ext}^{i+1}(M, N) .
$$

It quickly follows that the polynomials giving the lengths of $\operatorname{Ext}^{i}(M, N /$ $\left.I^{n} N\right)$ and $\operatorname{Ext}^{i+1}\left(M, I^{n} N\right)$ differ only by a constant and, consequently, have 
the same degree, assuming both lengths are not zero. We use this for the corollaries below.

In [6, page 763], it is asked whether, for fixed $i$, the polynomial giving the $i$ th Betti number of $I^{n} N$ has degree $\ell_{N}(I)-1$, provided it is not identically zero. One of the purposes of [5] was to show that the answer is yes in a number of cases. Similarly, by taking $M=\mathrm{k}$ in Theorem 4.7, we can now note that for fixed $i$ the polynomial giving the $i$ th Bass number of $I^{n} N$ is given by a polynomial of degree $d-1=\ell_{N}(I)-1$ in essentially the same cases.

Corollary 4.10. Let $(R, \mathfrak{m})$ be a local ring of dimension $d$, and let $N$ be a finitely generated $R$-module such that $N_{P} \neq 0$, for all minimal primes $P$. Assume that $I \subseteq R$ satisfies $\ell(I)=d$ and any one of the conditions (i)(iii) from Theorem 4.7. Then for any $i>0$ with $i$ less than the projective dimension of $\mathrm{k}$, the ith Bass numbers of $N / I^{n} N$ and $I^{n} N$ are given by polynomials of degree $d-1$.

The following instance of Corollary 4.10 deserves special attention.

Corollary 4.11. Let $(R, \mathfrak{m})$ be a quasi-unmixed local ring, and let $I$ be an integrally closed $\mathfrak{m}$-primary ideal. Then for any $i>0$ with $i$ less than the projective dimension of $\mathrm{k}$, the ith Bass numbers of $R / I^{n}$ and $I^{n}$ are given by polynomials of degree $d-1$.

\section{§5. Appendix}

As mentioned in Section 3, our results concerning the degree of $\mathcal{E}_{M, N, \mathfrak{m}}^{i}(n)$ are closely related to the dimension of the corresponding syzygies associated with $M$. As a consequence, we can shed some light on the following interesting question.

Question 5.1. Let $(R, \mathfrak{m})$ be a local ring, and let $M$ be a finitely generated $R$-module. Is the dimension of the $n$th syzygy $\Omega_{R}^{n}(M)$ stable for $n$ sufficiently large?

In the following remark, we record a few easy observations concerning Question 5.1.

REMARK 5.2. (i) If $R$ is unmixed and equidimensional, then clearly all syzygies have dimension equal to $\operatorname{dim}(R)$. 
(ii) If the dimension of syzygies is ultimately constant, then that constant value must equal $\operatorname{dim}(R)$. This follows since given two consecutive syzygies, one of them must have dimension equal to $\operatorname{dim}(R)$.

(iii) If $\beta_{i}(M)>\beta_{i-1}(M)$, then $\operatorname{dim}\left(\Omega_{R}^{i+1}(M)\right)=\operatorname{dim}(R)$. This is because, for any prime ideal $P,\left(\phi_{i}\right)_{P}$ cannot be injective. Thus, in fact, $\Omega_{R}^{i+1}(M)_{P} \neq 0$ for all $P$, so in this case $\Omega^{i+1}(M)$ has nilpotent annihilator. It follows that if the Betti numbers of $M$ are eventually increasing, then Question 5.1 has a positive answer for $M$.

(iv) If $\beta_{i}(M)<\beta_{i-1}(M)$, then $\operatorname{dim}\left(\Omega^{i-1}(M)\right)=\operatorname{dim}(R)$. This is because, for any prime ideal $P,\left(\phi_{i}\right)_{P}$ cannot be surjective. Thus, in fact, $\Omega^{i-1}(M)_{P} \neq$ 0 for all $P$, so in this case, $\Omega^{i-1}(M)$ has nilpotent annihilator.

Proposition 5.3. Let $(R, \mathfrak{m})$ be a local ring of dimension $d$, and let $M$ be a finitely generated $R$-module. Let $\Omega_{R}^{i+1}(M)$ be an $(i+1)$ st syzygy of $M$ with $1 \leq i<\operatorname{p.d} .(M)$. Assume that $\beta_{i}(M)=\beta_{i-1}(M)$. Then

$$
\max \left\{\operatorname{dim}\left(\operatorname{Ext}^{i}(M, R)\right), \operatorname{dim}\left(\Omega_{R}^{i+1}(M)\right)-1\right\} \geq d-2 .
$$

In particular, if $\operatorname{dim}(R) \geq 2$ and $M$ is free of constant rank on the punctured spectrum of $R$, then, in fact, $\operatorname{dim}\left(\Omega_{R}^{i+1}(M)\right)=d$.

Proof. We may assume that $\Omega_{R}^{i+1}(M)=\operatorname{im}\left(\phi_{i+1}\right)$. By Theorem 3.2,

$$
\operatorname{deg}\left(\mathcal{E}_{M, R, \mathfrak{m}}^{i}(n)\right)=\max \left\{\operatorname{dim}\left(\operatorname{Ext}^{i}(M, R)\right), \operatorname{dim}\left(T_{i}\right)-1\right\},
$$

where $T_{i}$ now denotes the image of the transpose of $\phi_{i+1}$ in $R^{\beta_{i+1}(M)}$. On the other hand, $\operatorname{dim}\left(T_{i}\right)=\operatorname{dim}\left(\Omega_{R}^{i+1}(M)\right)$, since for any prime $P$ the matrix $\left(\phi_{i+1}\right)_{P}$ is the zero matrix if and only if its transpose is the zero matrix. Therefore,

$$
\operatorname{deg}\left(\mathcal{E}_{M, R, \mathfrak{m}}^{i}(n)\right)=\max \left\{\operatorname{dim}\left(\operatorname{Ext}^{i}(M, R)\right), \operatorname{dim}\left(\Omega_{R}^{i+1}(M)\right)-1\right\} .
$$

The first statement in the corollary now follows from Corollaries 3.5(c) and 3.7 .

To prove the second statement, we note that $\operatorname{dim}\left(\operatorname{Ext}^{i}(M, R)\right)=0$ since $M$ is free on the punctured spectrum. Therefore, if $d \geq 3$, the maximum value above is $\operatorname{dim}\left(\Omega_{R}^{i+1}(M)\right)-1$. If $d=2$, the maximum value is again $\operatorname{dim}\left(\Omega_{R}^{i+1}(M)\right)-1$. For this, it is enough to show that the dimension of $\Omega_{R}^{i+1}(M)$ is positive. Assume, by way of contradiction, that $\operatorname{dim}\left(\Omega_{R}^{i+1}(M)\right)=$ 0 . Consider the exact sequence

$$
0 \rightarrow \Omega_{R}^{i+1}(M) \rightarrow R^{\beta_{i}(M)} \stackrel{\phi_{1}}{\rightarrow} R^{\beta_{i-1}(M)} \rightarrow \Omega_{R}^{i-1}(M) \rightarrow 0,
$$


where $\beta_{i}(M)=\beta_{i-1}(M)$. Since $\Omega_{R}^{i+1}(M)$ has finite length and $\Omega_{R}^{i-1}(M)$ is free of constant rank on the punctured spectrum, it follows from the sequence above that $\Omega_{R}^{i-1}(M)$ also has finite length. Thus, the $\operatorname{det}\left(\phi_{i}\right)$ generates an $\mathfrak{m}$-primary ideal, so $\operatorname{dim}(R) \leq 1$, which is a contradiction. Therefore, $\operatorname{dim}\left(\Omega_{R}^{i+1}(M)\right)$ is strictly positive and hence

$$
\operatorname{dim}\left(\Omega_{R}^{i+1}(M)\right)-1=\max \left\{\operatorname{dim}\left(\operatorname{Ext}^{i}(M, R)\right), \operatorname{dim}\left(\Omega_{R}^{i+1}(M)\right)-1\right\} \geq d-2,
$$

where the inequality follows from the first statement. Thus, $\operatorname{dim}\left(\Omega_{R}^{i+1}(M)\right) \geq$ $d-1$. To improve this, note that for any prime ideal $P \neq \mathfrak{m}, \Omega_{R}^{i+1}(M)_{P}$ is a free $R_{P}$-module of rank independent of $P$. If this rank were zero, then $\Omega_{R}^{i+1}(M)$ would have finite length. But since $\operatorname{dim}\left(\Omega_{R}^{i+1}(M)\right) \geq 1$, this cannot be. Thus, the constant rank of each $\Omega_{R}^{i+1}(M)_{P}$ is not zero, so $\Omega_{R}^{i+1}(M)_{P}$ is not zero for all nonmaximal primes $P$. In particular, $\operatorname{dim}\left(\Omega_{R}^{i+1}(M)\right)=d$.

The following example shown to us by Hamid Rahmati shows that we cannot relax the hypothesis $\operatorname{dim}(R) \geq 2$ in the second statement of Proposition 5.3.

EXAMPLE 5.4. Let $R:=\mathrm{k}[[x, y]] /\left(x^{2}, x y\right)$. Let $M:=R /(y)$, so that $M$ is a finite-length $R$-module. Consider the start of a free resolution of the $R$-module $M$

$$
0 \rightarrow x R \rightarrow R \stackrel{\cdot y}{\rightarrow} R \rightarrow M \rightarrow 0 .
$$

Since $x$ is a socle element, the second syzygy of $M$ also has finite length, and so $\operatorname{dim}\left(\Omega_{R}^{2}(M)\right)<\operatorname{dim}(R)$.

Proposition 5.5. Let $(R, \mathfrak{m})$ be a local ring, and let $M$ be a finitely generated $R$-module.

(a) If $M$ has nondecreasing Betti numbers, then $M$ has at most one syzygy $\Omega_{R}^{j}(M)$ with finite length. Moreover, $1 \leq j \leq d$.

(b) If the Betti numbers of $M$ are eventually nondecreasing, then $M$ has only finitely many syzygies with finite length.

Proof. For (a), suppose that $M$ has nondecreasing Betti numbers and that $M^{\prime}:=\Omega_{R}^{j}(M)$ is a syzygy of finite length. Then $M^{\prime}$ is free of constant rank zero on the punctured spectrum of $R$, so by Proposition 5.3, $\operatorname{dim}\left(\Omega_{R}^{i+1}\left(M^{\prime}\right)\right)=d$, for all $i \geq 1$. Thus, $\operatorname{dim}\left(\Omega_{R}^{s}(M)\right)=d$, for all $s \geq j+2$. On the other hand, the exact sequence

$$
0 \rightarrow \Omega_{R}^{j+1}(M) \rightarrow F_{j} \rightarrow \Omega_{R}^{j}(M) \rightarrow 0
$$


shows that $\operatorname{dim}\left(\Omega_{R}^{j+1}(M)\right)=d$, so $\operatorname{dim}\left(\Omega_{R}^{s}(M)\right)=d$, for all $s \geq j+1$. Note that this argument now precludes the possibility of $\Omega_{R}^{i}(M)$ having finite length for some $i<j$, so $M$ has at most one syzygy of finite length. To see that $j \leq d$, suppose, to the contrary, that $j>d$. Since $\Omega_{R}^{j}(M)_{P}=0$, for all prime ideals $P \neq \mathfrak{m}$, p.d. $\left(M_{P}\right)<\infty$, for all primes $P \neq \mathfrak{m}$. It follows from this that $\Omega_{R}^{d-1}(M)$ is free on the punctured spectrum of $R$. Note that since $\Omega_{R}^{j}(M)$ has finite length and since $j>d$, working backward from $\Omega_{R}^{j}(M)$ we see that $\Omega_{R}^{d-1}(M)$ must also have constant rank on the punctured spectrum. If we now apply Proposition 5.3 to $\Omega_{R}^{d-1}(M)$, it follows that $\operatorname{dim}\left(\Omega_{R}^{j}(M)\right)=d$, and this is a contradiction. Thus, $j \leq d$, as required. This finishes the proof of Proposition 5.5(a).

Finally, Proposition 5.5(b) follows immediately from the proof of the first statement in Proposition 5.5(a).

REMARK 5.6. It is clear from Proposition 5.5 that Question 5.1 is related to a more important question, namely, whether every finitely generated module over an arbitrary local ring has the property that its Betti numbers are eventually nondecreasing. Suppose that this latter property were true for all local rings. Replacing the module $M$ by one of its large syzygies, one could assume that the Betti numbers of $M$ were nondecreasing. If $\Omega_{R}^{i}(M)$ were a syzygy of dimension less than $d$, then we could localize at prime $P$ minimal in its support. By Proposition 5.3 and Proposition 5.5, $\operatorname{dim}\left(\Omega_{R}^{s}(M)_{P}\right)=\operatorname{dim}\left(R_{P}\right)$, for all $s \geq i+1$. Thus, if $R$ were equidimensional, then $\Omega_{R}^{s}(M)$ would have dimension $d$, for all $s \geq i+1$, and the dimension of the syzygies of $M$ would stabilize.

\section{REFERENCES}

[1] A. Corso, C. Huneke, D. Katz, and W. Vasconcelos, Integral closure of ideals and annihilators of homology, Lect. Notes Pure Appl. Math. 227 (2005), 33-48.

[2] A. Crabbe and J. Striuli, Constructing big indecomposable modules, Proc. Amer. Math. Soc. 137 (2009), 2181-2189.

[3] S. Iyengar and T. Puthenpurakal, Hilbert-Samuel functions of modules over CohenMacaulay local rings, Proc. Amer. Math. Soc. 135 (2007), 637-648.

[4] S. Goto, F. Hayasaka, and R. Takahashi, On vanishing of certain Ext modules, J. Math. Soc. Japan 60 (2008), 1045-1064.

[5] D. Katz and E. Theodorescu, On the degree of Hilbert polynomials associated to the torsion functor, Proc. Amer. Math. Soc. 135 (2007), 3073-3082.

[6] V. Kodiyalam, Homological invariants of powers of an ideal, Proc. Amer. Math. Soc. 118 (1993), 757-764. 
[7] E. Theodorescu, Derived functors and Hilbert polynomials, Math. Proc. Cambridge Philos. Soc. 132 (2002), 75-88.

Andrew Crabbe

Department of Mathematics

Syracuse University

Syracuse, New York 13244-1150

USA

amcrabbe@syr.edu

Daniel Katz

Department of Mathematics

University of Kansas

Lawrence, Kansas 66045

USA

dlk@math.ku .edu

Janet Striuli

Department of Mathematics and Computer Science

Fairfield University

Fairfield, Connecticut 06824

USA

jstriuli@mail.fairfield.edu

Emanoil Theodorescu

Division of Statistics

Northern Illinois University

De Kalb, Illinois 60115

USA

theodore@math.niu.edu 